\title{
Zufallsbefund kleiner Nierentumor: mehr Biopsie, weniger Operation?
}

\author{
Incidental Small Renal Tumor: More Biopsy, Less Surgery?
}

\author{
Martin Spoendlin ${ }^{1}$ und Gernot Bonkat ${ }^{1,2}$
}

${ }^{1}$ Merian Iselin Klinik, Basel

2alta uro AG, Basel

In der Grundversorgung ist man aufgrund der besseren und öfter eingesetzten Bildgebung immer häufiger mit der Herausforderung von Zufallsbefunden unklarer Ätiologie und Dignität konfrontiert. Oft steht man im unangenehmen Dilemma zwischen eventueller Überdiagnostik und Übertherapie und dem Risiko, ein Malignom mit therapeutischer Konsequenz zu verpassen.

Die Arbeit von Saba et al. [1] in dieser «Praxis»-Ausgabe setzt sich mit dem Problem der Überdiagnostik und Übertherapie bei kleinen, mittels Bildgebung entdeckten Nierentumoren auseinander. Im englischen Sprachgebrauch werden Nierentumoren $\leq 4 \mathrm{~cm}$ als Small Renal Masses (SRM) bezeichnet. Saba et al. haben alle Resektate von in Computertomogramm oder Magnetresonanztomogramm malignomverdächtigen Nierentumoren des Universitätsspitals Zürich von 2006 bis 2014 hinsichtlich ihrer Grösse in der Schnittbildgebung sowie der definitiven Histologie retrospektiv analysiert. Von 404 Resektaten hatten $221(54,7 \%)$ einen Tumordurchmesser $\leq 4 \mathrm{~cm}$. Unter diesen 221 SRM waren $28 \%$ benigne Läsionen und 70,6\% Malignome (Rest unsicheres oder geringes Malignitätspotenzial). Von den malignen Tumoren hatten $74,4 \%$ aufgrund der Histologie eine günstigere und 25,6\% eine ungünstigere Prognose. Diese Zahlen entsprechen etwa denen anderer publizierter Serien.

Tumorbiopsien gehören bisher meist nicht routinemässig zur Abklärung von SRM. Dies steht im Gegensatz zu den meisten anderen Tumorarten. Saba et al. können aufzeigen, dass der Stellenwert der Tumornierenbiopsie bei SRM bei der Therapieentscheidung künftig überdacht werden sollte.

Die Autorinnen und Autoren führen aus, dass die Biopsie einer SRM heutzutage eine vertretbare Komplikationsrate und eine relevante diagnostische Aussagekraft hat. Ferner legen sie dar, dass eine gefürchtete Hauptkomplikation, eine Tumorstreuung im Stichkanal, ausserordentlich selten und bei Auftreten mit unklarer klinischer Relevanz einzuordnen ist. Im Gegensatz hierzu kann die Rate höhergradiger Komplikationen bei einer Operation, meist einer partiellen Nephrektomie, bis zu 5,5\% betragen.

Eine Hauptaussage der Arbeit von Saba et al. ist, dass Biopsien von SRM helfen können, die operative Übertherapie bei betagten und komorbiden Personen mit einge- schränkter Lebenserwartung und prognostisch günstigeren Malignomen zu reduzieren und sie im Rahmen einer «Active Surveillance» zu überwachen. Es wird betont, dass dabei eine engagierte Interdisziplinarität und eine kritische Hinterfragung der Histologie im Gesamtkontext gefordert ist. Von verschiedenen Fachgesellschaften wurden hierzu ausführliche Richtlinien erstellt [2-6].

Wie Saba et al. ebenfalls aufzeigen, kann die Biopsie von SRM auch helfen, jüngeren und gesunden Personen mit benigner Biopsiehistologie allenfalls eine Operation zu ersparen. Auch Richtlinien [2-6] setzen sich mit dem Vorgehen bei bioptisch benignen Läsionen auseinander. Dabei muss die Treffsicherheit der Biopsie im Vergleich zu den histologischen Diagnosen an Operationspräparaten berücksichtigt werden. In einer Metaanalyse, die auch Serien mit Tumoren $>4 \mathrm{~cm}$ einschloss, waren Sensitivität und Spezifität der Biopsien exklusive Feinnadelpunktionen (FNPs) bezüglich Erfassen von Malignität in den 17 Serien, die dies untersuchten, zwar gut, aber eben nicht ganz $100 \%$. Zehn dieser 17 Serien fanden Sensitivitäten von $100 \%$. Die übrigen sieben Arbeiten beschrieben Sensitivitäten, die etwas tiefer lagen. Die schlechteste Sensitivität lag bei $88 \%$. Nur drei dieser 17 Studien waren prospektiv. Die Konkordanz zwischen Biopsien inkl. FNPs und Operationspräparaten bezüglich Subtyp von Nierenzellkarzinomen betrug in dieser Metanalyse 90,3\% (96\% für Tumoren $\leq 4 \mathrm{~cm}$ ). Bezüglich Grading lag die Konkordanz bei Anwendung einer vierstufigen Graduierung bei 62,5\% $(66,7 \%$ für Tumoren $\leq 4 \mathrm{~cm})$ und bei einer zweistufigen Graduierung bei 86,5\%. FNPs schnitten schlechter ab als Biopsien [7].

Eine andere retrospektive Studie ergab eine Treffsicherheit bezüglich Erfassen von Malignität von 97,1\%, wobei in dieser Studie grösstenteils nur Personen mit maligner Biopsiehistologie operiert und somit zur Evaluation der Biopsietreffsicherheit einbezogen wurden. Von den 95 Personen mit benigner Biopsiehistologie wurden nur sechs operiert. Drei von diesen (bioptisch zwei Onkozytome und ein Angiomyolipom), also 50\%, hatten aufgrund des Operationspräparates dann aber ein Nierenzellkarzinom [8].

Diese Daten stammen vorwiegend aus retrospektiven Studien. Die drei prospektiven Serien, die in der genann- 
ten Metaanalyse eingeschlossen waren, zeigten eine Sensitivität von Biopsien exkl. FNPs bezüglich des Erfassens von Malignität von 92, 93 und 95\% [7]. Um diese Sensitivität der Biopsien besser beurteilen zu können, wären Daten aus prospektiven Studien notwendig, bei denen auch alle Personen mit benigner Biopsiehistologie operiert würden. Beim grundsätzlich hilfreichen Einbezug der Tumornierenbiopsie bei der Therapieentscheidung muss man sich also bewusst sein, dass eine benigne Tumorbiopsie ein Malignom nicht sicher ausschliesst.

Besonders wichtig ist dieser Punkt bei den Onkozytomen, den häufigsten benignen Nierentumoren. Bei Onkozytomen existieren Hybridtumoren mit Anteilen von Nierenzellkarzinomen, die in der Biopsie verpasst werden können [9-11]. Die Häufigkeit von Hybridtumoren bei sporadischen Onkozytomen wird in einer Serie mit 4,2\% angegeben [11] und in einer anderen bei nicht weiter spezifizierten Onkozytomen mit 6,3\% [12]. Bei hereditären Onkozytomvarianten sind Hybridtumoren häufiger als bei sporadischen [10]. Die Koexistenz von Onkozytomen und Nierenzellkarzinomen bei derselben Person nebeneinander wird mit 10 bis zu 32\% beschrieben [13]. Weiter kann, wie auch in der Arbeit von Saba et al. [1] ausgeführt wird, die Unterscheidung zwischen einem benignen Onkozytom und einem chromophoben Nierenzellkarzinom eine Herausforderung für Pathologinnen und Pathologen sein. Mittels Biopsie diagnostizierte onkozytäre Tumoren waren in einer Metaanalyse aufgrund des Operationspräparates nur in $64,6 \%$ wirklich Onkozytome, $4,2 \%$ waren andere benigne Läsionen, $25 \%$ Nierenzellkarzinome und 6,3\% Hybridtumoren [12]. Langzeitbeobachtungen von nicht operierten Onkozytomen gibt es nicht, sondern nur Verlaufsstudien über ca. drei Jahre [9, 10, 14, 15]. Bezüglich Nichtoperieren von bioptisch benignen SRM und damit bezüglich der Indikation von Tumornierenbiopsien generell bei Erkrankten ohne eingeschränkte Lebenserwartung gibt es daher auch kritische Stimmen [9]. Es bedarf unbedingt in jedem Einzelfall beim Therapieentscheid einer engen interdisziplinären Zusammenarbeit zwischen Urologie, interventioneller Radiologie und Pathologie. Die Aussagekraft von Biopsiehistologie und Bildgebung muss kritisch analysiert und hinterfragt werden, um nicht bei einem in der Biopsie nicht erfassten Malignom von einer Operation abzusehen.

Dasselbe gilt grundsätzlich auch für Angiomyolipome, die zweithäufigsten benignen Nierentumoren, wobei hier die Bildgebung bei der fettreichen Form einen höheren diagnostischen Stellenwert hat. Zur Treffsicherheit der Biopsie bezüglich der Diagnose Angiomyolipom und der Abgrenzung gegen Malignome gibt es weniger Daten als für Onkozytome [16-22].

Die Veröffentlichung von Saba et al. [1] liefert für die Grundversorgung relevante und wertvolle Informationen. Es ist hilfreich zu wissen, dass betagte und komorbide Patientinnen und Patienten mit eingeschränkter Lebenserwartung mit zufällig entdeckten, prognostisch günstigen malignen SRM durch eine Biopsie des Tumors im Vorfeld vor einer Intervention bewahrt werden können. Mögliche
Komplikationen, eine allfällige Verschlechterung der Nierenfunktion und die Unannehmlichkeiten können damit verhindert werden. Wichtig ist, dass die Therapieentscheidung immer individuell aufgrund der Gesamtsituation im Dialog zwischen Patient_in, Urolog_in, Haus_ärztin und Patholog_in unter Berücksichtigung aktueller Richtlinien getroffen wird. Neben Tumorcharakteristika, Tumorgrösse, sowie der Effektivität und dem Komplikationspotenzial der verschiedenen Behandlungsmodalitäten müssen u.a. Alter, Lebenserwartung, Komorbiditäten und Nierenfunktion mitberücksichtigt werden. Die eingeschränkte Treffsicherheit der Biopsie bezüglich Tumorsubtyp und Grading, also bezüglich der Beurteilung, ob es sich wirklich um eine prognostisch eher günstigere Malignomform handelt, ist dabei zu berücksichtigen [7, 23]. Der Stellenwert der Tumornierenbiopsie mit benigner Histologie bei der Therapieentscheidung bei Patientinen und Patienten mit nicht eingeschränkter Lebenserwartung muss in jedem Einzelfall kritisch hinterfragt werden. Falls nicht umgehend operiert wird, ist in eine «Active Surveillance» indiziert.

\section{Bibliografie}

1. Saba K, Högger DC, Hötker AM, Rupp NJ, Sulser T, Hermanns T. Dignität kleiner Nierentumoren: Implikationen zur Abklärung und Therapie. Praxis. 2021;110:565-570.

2. Wilcox Vanden Berg RN, Basourakos SP, LaRussa S, McClure TD. Management of the small renal mass: a 2020 Update. Curr Oncol Rep. 2020;22(7):69.

3. Finelli A, Ismaila N, Bro B, et al. Management of small renal masses: American Society of Clinical Oncology Clinical Practice Guideline. J Clin Oncol. 2017;35(6):668-680.

4. Ljungberg B, Albiges L, Abu-Ghanem Y, et al. European Association of Urology Guidelines on renal cell carcinoma: The 2019 Update. Eur Urol. 2019;75(5):799-810.

5. Ljungberg B, Albiges L, Bedke J, et al. EU Guidelines on renal cell carcinoma. European Association of Urology 2021. https:// uroweb.org/wp-content/uploads/EAU-Guidelines-on-RenalCell-Carcinoma-2021.pdf; letzter Zugriff: 27.04.2021

6. Campbell S, Uzzo RG, Allaf ME, et al. Renal mass and localized renal cancer: AUA Guideline. J Urol. 2017;198(3):520-529.

7. Marconi L, Dabestani S, Lam TB, et al. Systematic review and meta-analysis of diagnostic accuracy of percutaneous renal tumour biopsy. Eur Urol. 2016;69(4):660-673.

8. Jeon HG, Seo SI, Jeong BC, et al. Percutaneous kidney biopsy for a small renal mass: a critical appraisal of results. J Urol. 2016;195(3):568-573.

9. Kutikov A, Smaldone MC, Uzzo RG, Haifler M, Bratslavsky G, Leibovich BC. Renal mass biopsy: always, sometimes, or never? Eur Urol. 2016;70(3):403-6.

10. Shuch B, Amin A, Armstrong AJ, et al. Understanding pathologic variants of renal cell carcinoma: distilling therapeutic opportunities from biologic complexity. Eur Urol. 2015;67(1):85-97.

11. Ginzburg S, Uzzo R, Al-Saleem T, et al. Coexisting hybrid malignancy in a solitary sporadic solid benign renal mass: implications for treating patients following renal biopsy. J Urol. 2014; 191(2):296-300.

12. Patel HD, Druskin SC, Rowe SP, Pierorazio PM, Gorin MA, Allaf ME. Surgical histopathology for suspected oncocytoma on renal mass biopsy: a systematic review and meta-analysis. BJU Int. 2017;119(5):661-666.

13. Abdessater M, Kanbar A, Comperat E, et al. Renal oncocytoma: an algorithm for diagnosis and management. Urology. 2020;143:173-180. 
14. Richard PO, Jewett MA, Bhatt JR, Evans AJ, Timilsina N, Finelli A. Active surveillance for renal neoplasms with oncocytic features is safe. J Urol. 2016;195(3):581-586.

15. Liu S, Lee S, Rashid P, et al. Active surveillance is suitable for intermediate term follow-up of renal oncocytoma diagnosed by percutaneous core biopsy. BJU Int. 2016;118(Suppl 3):30-34.

16. Jinzaki M, Silverman SG, Akita H, Mikami S, Oya M. Diagnosis of renal angiomyolipomas: classic, fat-poor, and epithelioid types. Semin Ultrasound CT MR. 2017;38(1):37-46.

17. Jinzaki M, Silverman SG, Akita H, Nagashima Y, Mikami S, Oya M. Renal angiomyolipoma: a radiological classification and update on recent developments in diagnosis and management. Abdom Imaging. 2014;39(3):588-604.

18. Allgood E, Raman SS. Image interpretation: practical triage of benign from malignant renal masses. Radiol Clin North Am. 2020;58(5):875-884

19. Nicolau C, Antunes N, Paño B, Sebastia C. Imaging characterization of renal masses. Medicina (Kaunas). 2021;8;57(1):51.

20. Kiefer RM, Stavropoulos SW. The role of interventional radiology techniques in the management of renal angiomyolipomas. Curr Urol Rep. 2017;18(5):36.

21. Herts BR, Silverman SG, Hindman NM, et al. Management of the incidental renal mass on CT: A White Paper of the ACR Incidental Findings Committee. J Am Coll Radiol. 2018;15(2):264273.

22. Park BK. Renal Angiomyolipoma: Radiologic classification and imaging features according to the amount of fat. AJR Am J Roentgenol. 2017;209(4):826-835
23. Ficarra V, Brunelli M, Novara G, et al. Accuracy of on-bench biopsies in the evaluation of the histological subtype, grade, and necrosis of renal tumours. Pathology. 2011;43(2):149-155.

English version online under DOI 10.1024/1661-8157/a003719

\section{Dr. med. Martin Spoendlin}

Nephrologie FMH und Allgemeine Innere Medizin FMH Merian Iselin Klinik

Föhrenstrasse 2

4054 Basel

martin.spoendlin@merianiselin.ch

\section{PD Dr. med. Gernot Bonkat}

Belegarzt an der Merian Iselin Klinik

alta uro $A G$

Centralbahnplatz 6

4051 Basel

bonkat@alta-uro.com 